\title{
Genotype-phenotype correlations in nonlethal osteogenesis imperfecta caused by mutations in the helical domain of collagen type I
}

\begin{abstract}
Frank Rauch ${ }^{\star, 1}$, Liljana Lalic ${ }^{1}$, Peter Roughley ${ }^{1}$ and Francis H Glorieux ${ }^{1}$
Osteogenesis imperfecta $(\mathrm{OI})$ is a heritable disorder with bone fragility that is often associated with short stature, tooth abnormalities (dentinogenesis imperfecta), and blue sclera. The most common mutations associated with Ol result from the substitution for glycine by another amino acid in the triple helical domain of either the $\alpha 1$ or the $\alpha 2$ chain of collagen type I. In this study, we compared the results of genotype analysis and clinical examination in 161 Ol patients (median age: 13 years) who had glycine mutations in the triple helical domain of $\alpha 1(I)(n=67)$ or $\alpha 2(I)(n=94)$. Serine substitutions were the most frequently encountered type of mutation in both chains. Compared with patients with serine substitutions in $\alpha 2(I)(n=40)$, patients with serine substitutions in $\alpha 1(\mathrm{I})(n=42)$ on average were shorter (median height $z$-score -6.0 vs $-3.4 ; P=0.005$ ), indicating that $\alpha 1(\mathrm{I})$ mutations cause a more severe phenotype. Height correlated with the location of the mutation in the $\alpha 2(\mathrm{I})$ chain but not in the $\alpha 1(\mathrm{I})$ chain. Patients with mutations affecting the first 120 amino acids at the amino-terminal end of the collagen type I triple helix had blue sclera but did not have dentinogenesis imperfecta. Among patients from different families sharing the same mutation, about 90 and $75 \%$ were concordant for dentinogenesis imperfecta and blue sclera, respectively. These data should be useful to predict disease phenotype in newly diagnosed 01 patients.
\end{abstract} European Journal of Human Genetics (2010) 18, 642-647; doi:10.1038/ejhg.2009.242; published online 20 January 2010

Keywords: COL1A1; COL1A2; collagen type I; genotype-phenotype; OI

\section{INTRODUCTION}

Osteogenesis imperfecta (OI) is a heritable connective tissue disorder that is mainly characterized by bone fragility and often short stature. Extraskeletal findings, such as tooth abnormalities (dentinogenesis imperfecta), blue or gray color of sclera, and, in adult years, hearing loss can be associated to a variable degree. The clinical spectrum represents a continuum ranging from perinatal lethality to nearly asymptomatic individuals with occasional fractures and normal stature. ${ }^{1}$

The large majority of individuals with a clinical diagnosis of OI have an identifiable mutation in COL1A1 or COL1A2, the genes that encode the two collagen type I alpha chains, $\alpha 1(\mathrm{I})$ and $\alpha 2(\mathrm{I}) .{ }^{1}$ OI patients with collagen type I mutations can be classified into four clinically defined types. ${ }^{2}$ OI type I comprises patients with absence of bone deformities and normal or near normal stature. Type II is lethal in the perinatal period. OI type III is the most severe form in children surviving the neonatal period and leads to extreme short stature. Patients with mild to moderate bone deformities and variable short stature are classified as OI type IV.

Both the $\alpha 1(\mathrm{I})$ and $\alpha 2(\mathrm{I})$ chains contain a central triple helical domain of 1014 amino acids. These triple helical domains are composed of uninterrupted repeats of the Gly-X-Y tripeptide, which are flanked by propeptides at both the amino- and carboxyl-terminal ends. During the assembly of collagen type I molecules, type I procollagen chains form a heterotrimer consisting of two pro $\alpha 1$ (I) chains and one pro $\alpha 2(\mathrm{I})$ chain. The chains first associate in the carboxyl-terminal propeptides and nucleate the triple helix, which is then propagated linearly toward the amino-terminal ends of the chains. Triple helix formation can only proceed normally if a glycine residue is present in every third position of the chains, because glycine is the only residue small enough to fit into the restricted space at the inside of the helix.

The most common mutations associated with OI result from the substitution for glycine by another amino acid in the triple helical domain of either the $\alpha 1$ (I) or the $\alpha 2$ (I) chain. More than 700 glycine mutations have been identified in OI patients and are listed in a database of type I collagen mutations (http://www.le.ac.uk/ge/ collagen/). ${ }^{3,4}$ Nevertheless, information on the correlation between genotype and phenotype is limited, mainly because the reported phenotypic information is sketchy. A few mutation reports have presented basic clinical characteristics, such as height, the presence of blue sclera or dentinogenesis imperfecta, and correlated these data to COL1A1 and COL1A2 sequencing results, but to our knowledge, none of these reports has described more than 20 OI patients with mutations in the triple helical domain of collagen type I. A compilation of 682 collagen type I triple helical mutations has recently been published, but the phenotypic information was limited to whether the patient survived the immediate postnatal period or not. ${ }^{5}$

In this study, we therefore aimed at providing a more detailed picture of genotype-phenotype correlations in OI patients with glycine mutations in the triple helical domain of collagen type I. Clinical data that are routinely collected during the assessment of OI

\footnotetext{
${ }^{1}$ Genetics Unit, Shriners Hospital for Children, Montreal, QC, Canada

*Correspondence: Dr F Rauch, Genetics Unit, Shriners Hospital for Children, 1529 Cedar Avenue, Montréal, Québec, Canada H3G 1 A6. Tel: +1 5148425964 ; Fax: +1 514842 5581; E-mail: frauch@shriners.mcgill.ca

Received 2 September 2009; accepted 16 December 2009; published online 20 January 2010
} 
Table 1 Phenotypic characteristics in patients with glycine substitutions in COL1A1 and COL1A2

\begin{tabular}{|c|c|c|c|c|c|c|c|}
\hline & $\mathrm{n}$ & All & $\mathrm{n}$ & COLIA1 & $\mathrm{n}$ & COL1A2 & P-value \\
\hline $\operatorname{Sex}(m / f)$ & 161 & $80 / 81$ & 67 & $33 / 34$ & 94 & $47 / 47$ & 0.93 \\
\hline OI type (I/III/IV) & 161 & $30 / 59 / 72$ & 67 & $11 / 29 / 27$ & 94 & $19 / 30 / 45$ & 0.34 \\
\hline Deformity or fracture at birth ( $n$ positive, \%) & 117 & $84(72)$ & 48 & $35(73)$ & 69 & $49(71)$ & 0.82 \\
\hline Blue sclerae ( $n$ positive, $\%$ ) & 150 & $96(64)$ & 59 & $44(75)$ & 91 & $52(57)$ & 0.03 \\
\hline Dentinogenesis imperfecta ( $n$ positive, \%) & 148 & $101(68)$ & 59 & $42(71)$ & 89 & $59(66)$ & 0.53 \\
\hline
\end{tabular}

Abbreviations: $f$, female; $m$, male; OI, osteogenesis imperfecta.

Shown are the number $(n)$ or, for $Z$-scores, median (interquartile range). The $P$-value indicates the significance of the difference between patients with $C O L 1 A 1$ and $C O L 1 A 2$ mutations ( $\chi^{2}$ - or U-test, as appropriate).

are presented on 161 patients who were examined at a single center over a 12-year period.

\section{SUBJECTS AND METHODS}

\section{Subjects}

This study includes patients who were evaluated at the Shriners Hospital for Children in Montreal between 1996 and 2008. Patients were assessed clinically by one of the authors (FR or FHG). When a diagnosis of OI was considered likely, DNA was obtained for sequence analysis of the COL1A1 and COL1A2 genes. The evaluation was also offered to those parents of pediatric OI patients who had OI themselves.

Owing to the study setting in a specialized pediatric orthopedic hospital, the study population only includes patients who had survived the immediate postnatal period and did not need to be treated in an intensive care unit. This series therefore includes patients who were diagnosed as having OI type I, III, or IV according to the Sillence classification, ${ }^{1}$ but not patients with the most severe phenotype (OI type II).

This analysis comprises all subjects in whom sequence analysis revealed abnormalities leading to the substitution of a glycine residue in the triple helical domain of the $\alpha 1$ (I) or $\alpha 2$ (I) chains by another amino acid. In total, 161 such patients were identified. Clinical characteristics (history of fractures or deformities at birth, height, weight, OI type, scleral hue, presence of dentinogenesis imperfecta) of these patients were extracted from their medical records by retrospective chart review. Information on hearing loss was not available, as audiometric testing was not part of the routine diagnostic workup.

As the clinical documentation was not always complete, the number of available phenotypic observations for each clinical characteristic can differ from the total patient number. The study was approved by the Shriners Hospital Institutional Review Board. Informed consent was obtained from the legal guardians and/or patients.

\section{Collagen type I mutation analysis}

Total genomic DNA was isolated from peripheral blood using the QIAamp DNA Blood Midi Kit (Qiagen Inc., Mississauga, ON, Canada). All exons of the COL1A1 and COL1A2 genes, including the exon-intron boundaries, were amplified by PCR using the primers previously described. ${ }^{6}$ The sequencing reaction was performed using a BigDye Terminator cycle sequencing kit (Applied Biosystems, Foster City, CA, USA). The nucleotide sequence was determined using an Applied Biosystems 3100 DNA sequencer.

Sequence traces were aligned with the GenBank reference sequences of the COL1A1 genomic DNA (AF017178) and CDNA (NM_000088.3), and the COL1A2 genomic DNA (AF004877.1) and cDNA (NM_000089.3). Collagen mutations are numbered following the convention (http://www.hgvs.org/ mutnomen/recs.html), which starts with the translation initiator methionine as amino acid +1 , and the A of the ATG codon as nucleotide +1 . In addition, triple helix positions are provided for amino acids within the triple helical region of each $\alpha(\mathrm{I})$ chain (residues encoded by codons 179-1192 of the COL1A1 transcript and 91-1104 of COL1A2).

A total of 44 of the mutations presented here had previously been included in a brief mutation report, ${ }^{7}$ a large compilation of OI mutations, ${ }^{5}$ a study on material bone density in $\mathrm{OI}^{8}$ and a small case series. ${ }^{9}$ The other mutations included in this report have been entered into a database of OI mutations (http://www.le.ac.uk/ge/collagen/). ${ }^{3,4}$ Mutations for which there was no prior entry in this database were labeled as 'novel.'

\section{Statistical analysis}

Height and weight measurements were converted to age- and sex-specific $z$-scores on the basis of reference data published by the Centers for Disease Control and Prevention. ${ }^{10}$ Group differences in dichotomous variables were tested for significance using the $\chi^{2}$-test. As most of the variables were not normally distributed, Mann-Whitney $U$-tests were used to evaluate group differences in continuous variables. Associations are given as Spearman's rank correlation. Phenotypic concordance between patients carrying recurrent mutation was evaluated by comparing the data of a randomly selected member of each family with a recurring mutation. The SDs of height $z$-scores between patients independently sharing the same mutation were calculated for each mutation. The averages of these SDs were calculated separately for the $\alpha 1$ (I) and the $\alpha 2$ (I) chains. A 5\% significance level was maintained throughout these analyses, and all tests were two-sided. Calculations were performed using SPSS software version 11.5 for Windows (SPSS, Inc., Chicago, IL, USA).

\section{RESULTS}

COL1A1 or COL1A2 mutations leading to substitutions for glycine in the collagen type I triple helix were found in 161 OI patients from 145 families. There were 111 distinct mutations, of which 38 affected the $\alpha 1$ (I) chain and 73 the $\alpha 2$ (I) chain. Altogether, 25 of the mutations were novel: 5 in COL1A1 and 20 in COL1A2. Overall, the majority of patients had a phenotypic diagnosis of OI type III or IV, had dentinogenesis imperfecta and blue sclera, and were born with skeletal deformities or fractures (Table 1). The median age was 13.0 years (range $0.3-50.9$ years) at the time of phenotypic analyses.

Clinical characteristics were largely similar between the $\alpha 1$ (I) and the $\alpha 2$ (I) mutation groups, but blue sclera were more frequently noted in patients with $\alpha 1$ (I) mutations than in patients with $\alpha 2$ (I) mutations. To eliminate the influence of different amino acid substitution patterns in $\alpha 1(\mathrm{I})$ and $\alpha 2(\mathrm{I})$, we repeated the comparison between chains for patients with the most prevalent type of mutation, serine substitutions. Compared with patients with serine substitutions in $\alpha 2$ (I) $(n=40)$, patients with serine substitutions in $\alpha 1(\mathrm{I})(n=42)$ on average were shorter (median height $z$-score $-6.0 v s-3.4 ; P=0.005$ ), and a higher proportion had a diagnosis of OI type III (59 vs 25\%; 
Table 2 Effect of specific amino-acid residue substitutions for glycine in $\alpha 1$ (I)

\begin{tabular}{|c|c|c|c|c|c|c|c|c|}
\hline & \multicolumn{2}{|c|}{ Deformities or fractures at birth } & \multicolumn{2}{|c|}{ Blue sclera } & \multicolumn{2}{|c|}{ Dentinogenesis imperfecta } & \multicolumn{2}{|c|}{ Height (z-score) } \\
\hline & $\mathrm{n}$ & n positive (\%) & $\mathrm{n}$ & n positive (\%) & $\mathrm{n}$ & n positive (\%) & $\mathrm{n}$ & Median \\
\hline Ser & 30 & $25(83)$ & 35 & $23(66)$ & 36 & $32(89)$ & 42 & -6.0 \\
\hline Asp & 3 & $2(66)$ & 4 & $4(100)$ & 4 & $1(25)$ & 4 & -4.6 \\
\hline Cys & 3 & $1(33)$ & 3 & $2(67)$ & 3 & $3(100)$ & 3 & -1.9 \\
\hline Ala & 2 & $2(100)$ & 3 & $2(67)$ & 3 & $3(100)$ & 3 & -3.8 \\
\hline
\end{tabular}

Abbreviation: NA, not applicable.

The asterisks indicate significant differences $\left({ }^{*} P<0.05 ;{ }^{* * *} P<0.001\right)$ compared with patients with serine mutations $\left(U\right.$ - or $\chi^{2}$-test, as appropriate)

$P=0.002)$ and was positive for dentinogenesis imperfecta (89 vs $66 \%$; $P=0.02)$. No group differences were found in the prevalence of blue sclera and weight $z$-scores $(P=0.21)$.

\section{Glycine mutations in the $\alpha 1$ (I) chain}

Second to serine substitutions, arginine substitutions were the most common type of mutation affecting $\alpha 1$ (I) (Table 2). Substitutions by other amino acids were rare. With the exception of one arginine substitution at triple helical Gly667 (p.Gly845), substitutions by amino acids with branched or charged side chains (arginine, aspartate, glutamate, valine) were found only in the first 154 amino-terminal amino acid residues of the $\alpha 1$ (I) triple helical domain (p.Gly179 to p.Gly332). Compared with patients with serine substitutions, patients with arginine substitutions were significantly taller and a smaller percentage of patients with arginine substitutions had fractures or deformities at birth or had dentinogenesis imperfecta (Table 2). The phenotypic effects of substitutions by amino acids other than arginine could not be evaluated in a statistically meaningful manner because of insufficient sample size.

The variation of phenotype with the position of a mutation is shown in Figures 1 and 2. There are large gaps in the mutation distribution, which in part correspond to the 'exclusively lethal regions' (helix positions 691-823 and 910-964) described by Marini et al. ${ }^{5}$ However, one patient (OI type IV, final height $152 \mathrm{~cm}$, able to walk independently) had a mutation (a substitution of alanine for glycine at helical position 955 (p.Gly1133Ala) in one of these 'exclusively lethal regions.'

The position of glycine mutations within the $\alpha 1(\mathrm{I})$ triple helical domain had no obvious relationship with the presence of fractures or deformities at birth (Figure 1). However, scleral hue seemed to vary with the site of a glycine mutation. All 16 patients with mutations amino-terminal of triple helical Gly154 (p.Gly332) had blue sclera, whereas 15 of the 43 patients (35\%) who had glycine substitutions in the remainder of the $\alpha 1$ (I) triple helical domain did not have blue sclera.

The dental phenotype also correlated with the position of the glycine mutation. None of the 15 patients with mutations aminoterminal of Gly127 (p.Gly305) had dentinogenesis imperfecta, whereas 49 out of 52 patients with mutations that were carboxy-terminal of Gly127 had dentinogenesis imperfecta.

Height $z$-scores did not correlate in a statistically significant manner with the location of the mutated residue in the $\alpha 1$ (I) triple helical domain (Figure 2). This was true both for the entire group of patients with $\alpha 1(\mathrm{I})$ mutations and for the subgroup with serine substitutions.
In the $\alpha 1(\mathrm{I})$ chain, 11 mutations recurred independently in two or more families. Among patients from different families sharing the same mutation in $\alpha 1(\mathrm{I}), 29$ of 31 patients (94\%) had concordance for dentinogenesis imperfecta, 22 of 29 (76\%) for blue sclera, and 18 of $24(75 \%)$ for fractures or deformities at birth. The average SD for height $z$-score among unrelated patients sharing the same mutation was 2.2 .

\section{Glycine mutations in the $\alpha 2(\mathrm{I})$ chain}

Similar to the $\alpha 1$ (I) chain, serine substitutions were the most frequent type of mutation affecting the triple helical domain of $\alpha 2$ (I) (Table 3). Next in order of frequency were aspartate, arginine, and cysteine substitutions. In contrast to $\alpha 1$ (I), substitutions for amino acids with branched or charged side chains were found in both the aminoterminal and the carboxy-terminal portions of the $\alpha 2$ (I) triple helical domain.

Patients with aspartate substitutions on average were shorter than patients with serine substitutions (Table 3 ). No statistically significant phenotypic differences were found between patients with arginine and serine substitutions. Substitutions by other amino acids were too rare for group comparisons.

The variation of phenotype with the position of a mutation is shown in Figures 1 and 2 . In the $\alpha 2$ (I) chain, stretches without mutations were less conspicuous than in the $\alpha 1$ (I) chain and in part corresponded to the 'lethal clusters' identified by Marini et al. However, six patients had mutations in one of these eight 'lethal clusters. One of these patients (mutation: p.Gly790Ala) was a boy with a diagnosis of mild OI type I and normal height $(170 \mathrm{~cm}$ at age 15 years). The other five patients (mutations: p.Gly571Arg, p.Gly664Asp, p.Gly715Ser, p.Gly955Ser, p.Gly982Ser) were girls with a diagnosis of OI type III $(n=3)$ or OI type IV $(n=2)$ who had severe short stature (height $z$-score between -4.6 and -13.9 ).

The position of glycine mutations within the $\alpha 2(\mathrm{I})$ triple helical domain had no obvious relationship with the presence of fractures or deformities at birth or with scleral hue (Figure 1). However, the prevalence of dentinogenesis imperfecta varied according to the position of the affected glycine residue (Figure 1). None of the six patients with mutations affecting the first 121 residues of the $\alpha 2$ (I) triple helical domain (p.Gly90 to p.Gly211) had dentinogenesis imperfecta. In contrast, all but 2 of the 28 patients with mutations affecting a glycine residue in the two stretches between Gly286 and Gly655 (p.Gly376 and p.Gly745) and from Gly892 (p.Gly982) to the amino-terminal end of the triple helical domain had dentinogenesis imperfecta.

In the entire group of patients with mutations in the $\alpha 2$ (I) triple helical domain, height $z$-scores correlated inversely with the position 
Alpha 1 (I)
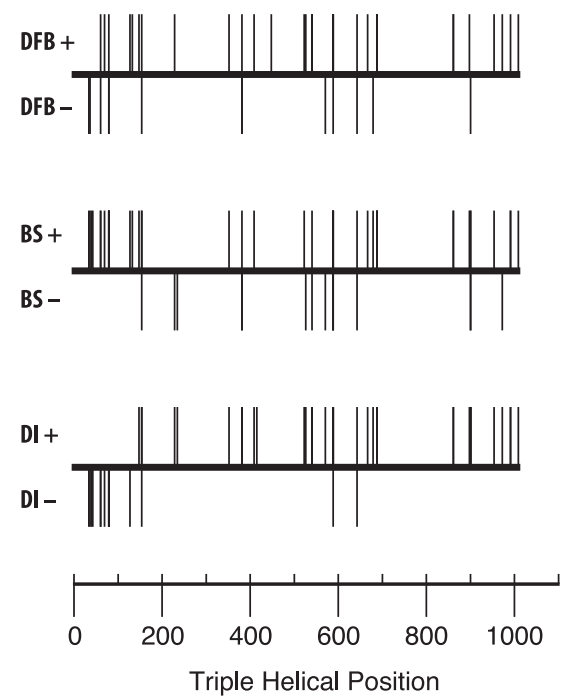

Alpha 2 (I)
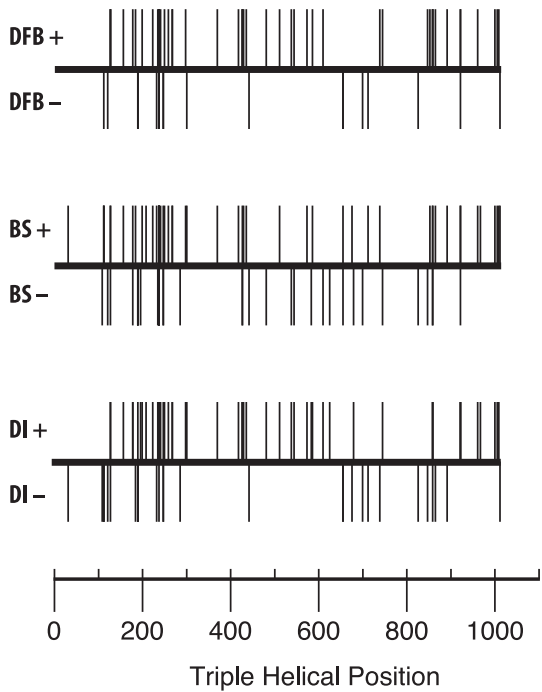

Figure 1 Relationship between the triple helical position of glycine mutations in collagen type I $\alpha$ chains and the presence (+) or absence (-) of deformities or fractures at birth (DFB), blue sclera (BS), and dentinogenesis imperfecta (DI).
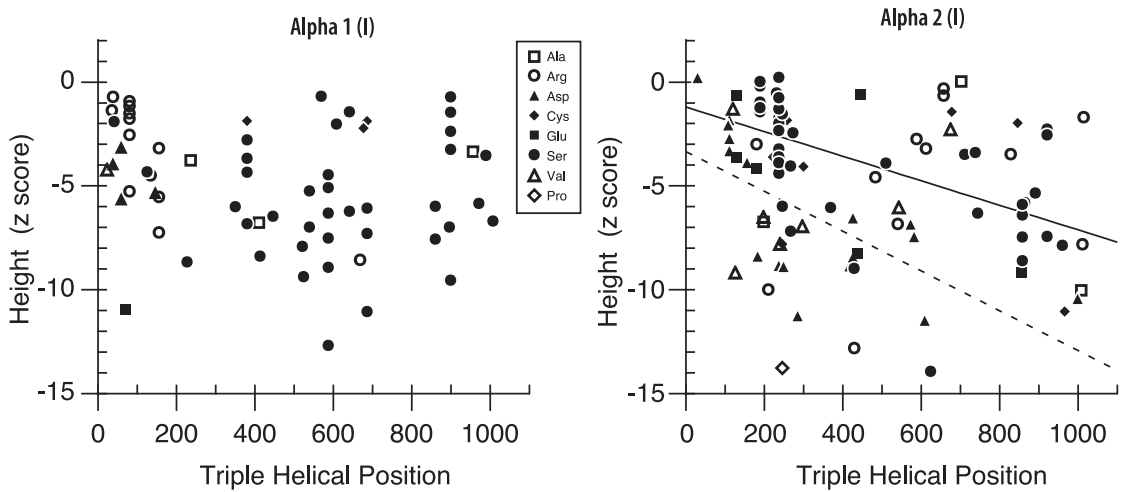

Figure 2 Relationship between the positions of glycine substitutions in the triple helical domain of type I collagen and height $z$-scores. The oblique solid lines represent the regression lines between the position of serine substitutions in $\alpha 2(\mathrm{I})$ and height $(r=-0.73, P<0.001$; Spearman's rank correlation). The oblique dashed line represents the regression line between the position of aspartate substitutions in $\alpha 2(I)$ and height $(r=-0.75, P<0.001)$.

Table 3 Effect of specific amino-acid residue substitutions for glycine in $\alpha 2(\mathrm{I})$

\begin{tabular}{|c|c|c|c|c|c|c|c|c|}
\hline & \multicolumn{2}{|c|}{ Deformities or fractures at birth } & \multicolumn{2}{|c|}{ Blue sclera } & \multicolumn{2}{|c|}{ Dentinogenesis imperfecta } & \multicolumn{2}{|c|}{ Height (z-score) } \\
\hline & $\mathrm{n}$ & n positive (\%) & $\mathrm{n}$ & n positive (\%) & $\mathrm{n}$ & n positive (\%) & $\mathrm{n}$ & Median \\
\hline Ser & 30 & $19(63)$ & 39 & $20(51)$ & 38 & $25(66)$ & 40 & -3.4 \\
\hline Arg & 10 & $6(60)$ & 11 & $6(55)$ & 11 & $7(64)$ & 12 & -3.3 \\
\hline Cys & 4 & $3(75)$ & 6 & $4(67)$ & 6 & $5(83)$ & 7 & -3.6 \\
\hline Ala & 2 & $1(50)$ & 3 & $1(33)$ & 3 & $2(67)$ & 3 & -6.7 \\
\hline Pro & 0 & 0 & 1 & $1(100)$ & 1 & $1(100)$ & 1 & -13.8 \\
\hline
\end{tabular}

The asterisks indicate significant differences $(* P<0.05)$ compared with patients with serine mutations ( $U$ - or $\chi^{2}$-test, as appropriate).

of the glycine mutation $(r=-0.33(P<0.001)$ ) (Figure 2). Similar associations were also found in the subgroups with serine and aspartate substitutions (Figure 2).

In the $\alpha 2(\mathrm{I})$ chain, seven mutations occurred in two or more families. Among patients from different families sharing the same mutation in $\alpha 2(\mathrm{I}), 22$ of 24 (92\%) had concordance for dentinogenesis imperfecta, 17 of $25(68 \%)$ for blue sclera, and 18 of $24(81 \%)$ for fractures or deformities at birth. The average $\mathrm{SD}$ for height $z$-scores among unrelated patients sharing the same mutation was 2.4 . 


\section{DISCUSSION}

This analysis considerably enlarges the knowledge base for the phenotypic consequences of glycine mutations in the triple helical domain of collagen type I. In a recent consortium effort, Marini et a $t^{5}$ compiled 682 such mutations from many different laboratories, but provided little phenotypic information. None of the mutation reports presenting more detailed clinical data has included more than 20 patients with collagen type I triple helical mutations.

Compared with the report by Marini et al, this study contains a relatively larger number of mutations affecting the $\alpha 2$ (I) chain. We found almost twice as many mutations in $\alpha 2$ (I) than in $\alpha 1$ (I), whereas the compilation of Marini et al contained slightly more 'nonlethal' $\alpha 1$ (I) than $\alpha 2$ (I) triple helical mutations. This discrepancy may partly be due to methodological differences in mutation detection. Relative mutation frequencies may also depend on what basis clinicians request collagen type I mutation analyses. At our institution, these analyses were performed not only in patients with the typical features of OI but also in cases of bone fragility where the clinical diagnosis was more uncertain. It is therefore possible that the patients of this study on average were less severely affected than the 'nonlethal cases' described by Marini et al.

Our observations were mostly compatible with the findings of Marini et al that mutations in some regions of the $\alpha 1$ (I) and $\alpha 2$ (I) chains are usually lethal. However, it should be noted that a total of seven patients in our series had mutations in such 'lethal' regions. Thus, although the identification of such areas is certainly very useful for elucidating the function of different regions of $\alpha(\mathrm{I})$ chains, clinicians must be aware that mutations in such areas can be compatible with long-term survival and sometimes even a mild phenotype.

Mutations affecting $\alpha 1(\mathrm{I})$ are thought to lead to a more severe phenotype than $\alpha 2$ (I) mutations, because the collagen type I heterotrimer consists of two $\alpha 1$ (I) chains but only one $\alpha 2$ (I) chain. ${ }^{11}$ In this study, phenotypic characteristics were similar between the entire group of patients with $\alpha 1$ (I) and those with $\alpha 2$ (I) mutations. However, the frequency of amino acid residues substituting for glycine differed between the $\alpha 1$ (I) and the $\alpha 2$ (I) chains. When the analysis was limited to patients with serine substitutions, it emerged that patients with mutations in $\alpha 1$ (I) on average were indeed more severely affected.

The study by Marini et al found that mutations in $\alpha 1$ (I) lead to a more variable outcome than $\alpha 2$ (I) mutations. ${ }^{5}$ In this study, however, the phenotypic concordance between patients with recurrent mutations seemed to be similar for $\alpha 1(\mathrm{I})$ and $\alpha 2(\mathrm{I})$ mutations. This difference between studies may be influenced by the fact that this study examined a smaller severity spectrum, as we examined only nonlethal mutations, whereas Marini et al examined both lethal and nonlethal mutations.

Our findings confirm earlier observations that nonlethal substitutions by amino acid residues with branched or charged side chains are mostly restricted to the amino-terminal portion of the $\alpha 1$ (I) triple helical domain, whereas such substitutions have lethal consequences in other parts of $\alpha 1$ (I). ${ }^{5,12}$ However, when they occur close to the aminoterminal end, the phenotypic consequences of substitutions by amino acids with branched or charged side chains can be relatively mild, as highlighted by our finding that patients with arginine substitutions on average were taller than those with serine substitutions.

In $\alpha 2(\mathrm{I})$, height $z$-scores were negatively correlated with the location of the mutated glycine residue, whereas no such association was found in $\alpha 1(\mathrm{I})$. Similar observations were made when these regression analyses were limited to serine substitutions. This suggests that in the $\alpha 2$ (I) chain, but not in the $\alpha 1$ (I) chain, mutations that are closer to the carboxy-terminal end of the triple helix tend to lead to a more severe skeletal phenotype. It has long been hypothesized that glycine substitutions toward the carboxy terminus of the $\alpha$-chains will be more clinically severe than those toward the amino terminus, consistent with the model that, as helix formation propagates from the carboxy terminus to the amino terminus, those mutations toward the carboxy terminus will be more disruptive to helix formation. ${ }^{13}$ The present data are compatible with this 'gradient model' of disease severity for $\alpha 2$ (I) mutations but not for mutations affecting $\alpha 1$ (I).

Our findings confirm previous observations that the majority of patients with glycine mutations in $\alpha 1$ (I) or $\alpha 2$ (I) have clinically recognizable dentinogenesis imperfecta. ${ }^{14}$ However, our data also suggest that dentinogenesis imperfecta is absent in patients who have mutations in the amino-terminal end of the $\alpha 1$ (I) or $\alpha 2$ (I) triple helical domain. In fact, none of the 21 patients with glycine mutations in the first 127 amino acids of the $\alpha 1$ (I) or the first 121 amino acids of the $\alpha 2$ (I) triple helical domain had been diagnosed with dentinogenesis imperfecta. This seems to be a novel observation, which however is in accordance with the existing literature. Various investigators have reported on the dental status of OI patients with glycine mutations in this region of the collagen type I triple helix and none of the nine patients mentioned in these reports had clear clinical evidence of dentinogenesis imperfecta. ${ }^{14-18}$

The mechanistic link between collagen type I mutations and dentinogenesis imperfecta has not been elucidated in any detail. Our data nevertheless show that patients sharing the same mutation had a high concordance for the condition. This suggests that dentinogenesis imperfecta is determined mostly by the type of collagen mutation and less by other genetic or environmental factors.

Patients with the same collagen type I mutation were less concordant for the presence of blue sclera. This may be related to the observation that the scleral hue of an OI patient is not constant and can normalize with age. ${ }^{19}$ Nevertheless, we found that all patients with mutations in the amino-terminal end of the $\alpha 1$ (I) chain had blue sclera. The mechanism for this genotype-phenotype correlation is unclear at present, as the pathophysiology of the scleral discoloration in OI has not been studied in detail and animal models are lacking.

In conclusion, this study shows genotype-phenotype correlations for 161 OI patients with 111 distinct triple helical mutations of collagen type I. These data should help to delineate the functional relevance of the various parts of the collagen type I triple helical domain and should also be useful to predict disease phenotype in newly diagnosed OI patients.

\section{CONFLICT OF INTEREST}

The authors declare no conflict of interest.

\section{ACKNOWLEDGEMENTS}

We thank Mark Lepik for the preparation of the figures. FR received salary support from the Chercheur-Boursier Clinicien program of the Fonds de la Recherche en Santé du Québec. This study was supported by the Shriners of North America.

1 Rauch F, Glorieux FH: Osteogenesis imperfecta. Lancet 2004; 363: 1377-1385.

2 Sillence DO, Senn A, Danks DM: Genetic heterogeneity in osteogenesis imperfecta. J Med Genet 1979; 16: 101-116.

3 Dalgleish R: The human type I collagen mutation database. Nucleic Acids Res 1997; 25: 181-187.

4 Dalgleish R: The human collagen mutation database 1998. Nucleic Acids Res 1998; 26: 253-255. 
5 Marini JC, Forlino A, Cabral WA et al: Consortium for osteogenesis imperfecta mutations in the helical domain of type I collagen: regions rich in lethal mutations align with collagen binding sites for integrins and proteoglycans. Hum Mutat 2007 , 28: 209-221

6 Korkko J, Ala-Kokko L, De Paepe A, Nuytinck L, Earley J, Prockop DJ: Analysis of the COL1A1 and COL1A2 genes by PCR amplification and scanning by conformationsensitive gel electrophoresis identifies only COL1A1 mutations in 15 patients with osteogenesis imperfecta type I: identification of common sequences of null-allele mutations. Am J Hum Genet 1998; 62: 98-110.

7 Ward LM, Lalic L, Roughley PJ, Glorieux FH: Thirty-three novel COL1A1 and COL1A2 mutations in patients with osteogenesis imperfecta types I-IV. Hum Mutat 2001; 17: 434.

8 Roschger P, Fratzl-Zelman N, Misof BM, Glorieux FH, Klaushofer K, Rauch F: Evidence that abnormal high bone mineralization in growing children with osteogenesis imperfecta is not associated with specific collagen mutations. Calcif Tissue Int 2008; 82: 263-270.

9 Faqeih E, Roughley P, Glorieux FH, Rauch F: Osteogenesis imperfecta type III with intracranial hemorrhage and brachydactyly associated with mutations in exon 49 of COL1A2. Am J Med Genet A 2009; 149A: 461-465.

10 Ogden CL, Kuczmarski RJ, Flegal KM et al: Centers for Disease Control and Prevention 2000 growth charts for the United States: improvements to the 1977 National Center for Health Statistics version. Pediatrics 2002; 109: 45-60.

11 Prockop DJ, Constantinou CD, Dombrowski KE et al: Type I procollagen: the geneprotein system that harbors most of the mutations causing osteogenesis imperfecta and probably more common heritable disorders of connective tissue. Am J Med Genet 1989; 34: 60-67.

12 Bodian DL, Chan TF, Poon A et al: Mutation and polymorphism spectrum in osteogenesis imperfecta type II: implications for genotype-phenotype relationships. Hum Mol Genet 2009; 18: 463-471.

13 Byers PH: Brittle bones - fragile molecules: disorders of collagen gene structure and expression. Trends Genet 1990; 6: 293-300.

14 Lund AM, Jensen BL, Nielsen LA, Skovby F: Dental manifestations of osteogenesis imperfecta and abnormalities of collagen I metabolism. J Craniofac Genet Dev Biol 1998; 18: 30-37

15 Starman BJ, Eyre D, Charbonneau $\mathrm{H}$ et al: Osteogenesis imperfecta. The position of substitution for glycine by cysteine in the triple helical domain of the pro alpha $1(\mathrm{I})$ chains of type I collagen determines the clinical phenotype. J Clin Invest 1989; 84: 1206-1214.

16 Valli M, Zolezzi F, Mottes M et al: Gly85 to Val substitution in pro alpha 1 (I) chain causes mild osteogenesis imperfecta and introduces a susceptibility to protease digestion. Eur J Biochem 1993; 217: 77-82.

17 Benusiene E, Kucinskas V: COL1A1 mutation analysis in Lithuanian patients with osteogenesis imperfecta. J App/ Genet 2003; 44: 95-102.

18 Venturi G, Tedeschi E, Mottes M et al: Osteogenesis imperfecta: clinical, biochemical and molecular findings. Clin Genet 2006; 70: 131-139.

19 Sillence D, Butler B, Latham M, Barlow K: Natural history of blue sclerae in osteogenesis imperfecta. Am J Med Genet 1993; 45: 183-186. 